\title{
Neoclassical Transport in Quasi-Axially Symmetric Stellarators
}

\author{
H.E.Mynick \\ Plasma Physics Laboratory, Princeton University \\ P.O. Box 451 \\ Princeton, New Jersey 08543-0451, U.S.A.
}

\begin{abstract}
We present a numerical and analytic assessment of the transport in two quasi-axially symmetric stellarators, including one variant of the $\mathrm{MHH} 2$ class[1] of such devices, and a configuration we refer to as $\mathrm{NHH} 2$, closely related to $\mathrm{MHH} 2$. Monte Carlo simulation results are compared with expectations from established stellarator neoclassical theory, and with some empirical stellarator scalings, used as an estimate of the turbulent transport which might be expected. From the standpoint of transport, these may be viewed as either tokamaks with large $(\delta \sim 1 \%)$ but low- $n$ ripple, or as stellarators with small ripple. For NHH2, numerical results are reasonably well explained by analytic neoclassical theory. MHH2 adheres less to assumptions made in most analytic theory, and its numerical results agree less well with theory than those for NHH2. However, for both, the non-axisymmetric contribution to the heat flux is comparable with the symmetric neoclassical contribution, and also falls into the range of the expected anomalous
\end{abstract}


(turbulent) contribution. Thus, it appears effort to further optimize the thermal transport beyond the particular incarnations studied here would be of at most modest utility. However, the favorable thermal confinement relies heavily on the radial electric field. Thus, the present configurations will have a loss cone for trapped energetic ions, so that further optimization may be indicated for large devices of this type.

\section{DISCLAIMER}

This report was prepared as an account of work sponsored by an agency of the United States Government. Neither the United States Government nor any agency thereof, nor any of their employees, makes any warranty, express or implied, or assumes any legal liability or responsibility for the accuracy, completeness, or usefulness of any information, apparatus, product, or process disclosed, or represents that its use would not infringe privately owned rights. Reference herein to any specific commercial product, process, or service by trade name, trademark, manufacturer, or otherwise does not necessarily constitute or imply its endorsement, recommendation, or favoring by the United States Government or any agency thereof. The views and opinions of authors expressed herein do not necessarily state or reflect those of the United States Government or any agency thereof. 


\section{DISCLAIMER}

Portions of this document may be illegible in electronic image products. Images are produced from the best available original document. 


\section{Introduction}

This work describes an assessment of the confinement characteristics of two related stellarators having quasi-axial (QA) symmetry . We present results for one variant of the $\mathrm{MHH} 2$ concept[1] - a 2-period $(N=2) \bmod$ ular QA stellarator, and a second system we refer to as NHH2, obtained from the MHH2 description, but modified in a simple manner (to be described shortly) which makes it conform better to usual assumptions made in existing stellarator transport theory, and therefore permits us to carry the analytic development further. The principal objective of such magnetic configurations is transport optimization, in this case by eliminating nonaxisymmetric components of the magnetic field strength $B(\psi, \theta, \zeta)$ in Boozer coordinates, i.e., those harmonics with $n \neq 0$ in the decomposition

$$
B(\psi, \theta, \zeta)=\sum_{m, n} B_{m n}(\psi) \cos (n \zeta-m \theta)
$$

Then the residual neoclassical transport is as in a tokamak. If the ideal of this configuration could be achieved, it would have the transport advantages of the equivalent tokamak, while retaining the stellarator virtues of needing no internal current to provide its rotational transform $\iota(\psi) \equiv q^{-1}(\psi)$, steady-state operation without current drive, and being more resistant to disruptions.

The particular variant of $\mathrm{MHH} 2$ studied here is an $N_{c}=16$ coil design, with major radius $R_{0}=3 \mathrm{~m}$, average minor radius $a=.66 \mathrm{~m}$, hence inverse apsect ratio $\epsilon_{a} \equiv a / R_{0}=1 / 4.5$, and average magnetic field on axis $B_{0}=2$ T. The shape of the outermost flux surface was specified by P. Garabedian, and the magnetic fields computed[2] using the VMEC code.[3] Thus, the ripple from the discreteness of the field coils is neglected in this study, and is 
expected not to produce appreciable transport except near the edge. NHH2 is a mathematical construct, having fields and other parameters precisely the same as in MHH2, but with the sign of $m$ reversed in each $B_{m n}$.

As desired, the average residual ripple strength $\delta$ in these systems is small in comparison with that typical of stellarators, which have $\delta \sim \epsilon \equiv r / R_{0}$, but is appreciable in comparison with that from coil disceteness in realistic tokamaks. Since large ripple $(\delta \sim 1 \%)$ can also be problematic in tokamaks, it is not $a$ priori clear that the systems considered here will have acceptably low neoclassical transport. Additionally, the $n$-value associated with the ripple here is about an order of magnitude lower than that normally envisioned for rippled tokamaks. Thus, MHH2 and NHH2 have features which are not strictly in accord with the assumptions made in existing transport theory for both stellarators and tokamaks. One result of this work is to provide some calibration of that theory with the $\mathrm{MHH} 2$ design. Especially important in these theories is a separation in length scales between the toroidal connection length $L_{t} \sim q R_{0}$ and the length $L_{r} \sim L_{t} /|n q-m|$ across a ripple well. The reversal of poloidal mode number $m$ in the $B_{m n}$ 's makes this separation better for NHH2. Thus one expects, and finds numerically, that transport in NHH2 should adhere better with existing theory than that in MHH2.

The remainder of the paper is organized as follows. In Sec. 2 we describe the character of the fields and particle orbits in them, and briefly review the theoretical transport results to which our numerical results will be compared. In Sec. 3 we present the numerical results, transport coefficients obtained from Monte Carlo (MC) simulations done with GC3, a guiding-center (GC) code in Boozer coordinates, and compare these with the analytical results already introduced. The ensembles used in the MC 
runs are for monoenergetic particle distributions, in order to obtain adequate statistics for ensembles of modest size. The approximate agreement between the analytic and numerical results for NHH2 shown in Sec. 3 permits us in Sec. 4 to perform a theory-based extension of the monoenergetic results to the 'energy-averaged' expressions applicable to a local Maxwellian distribution $f_{M}$, for the transport coefficients, particle and energy fluxes, and associated confinement times.

The ion fluxes depend sensitively on the radial electric field $E_{r} \simeq-\partial_{r} \Phi$ [with $\Phi(r$ or $\psi)$ the ambipolar potential and $r \equiv\left(2 \psi / B_{0}\right)^{1 / 2}$ a flux function with units of length, representing the average minor radius], and these analytic expressions for the fluxes put us in a position to determine those values of $E_{r}$ which satisfy the ambipolarity constraint

$$
0=\sum_{s} Z_{s} \Gamma_{1 s}
$$

where $s$ is a species label, $Z_{s} \equiv e_{s} / e_{0}$ is the charge number for species $s$, $e_{0}$ is the proton charge, and $\Gamma_{1 s}$ is the particle flux for species $s$. Here, we as usual consider a 2-species plasma, electrons and a single ion species. As found over a decade ago[4,5], for some parameters multiple roots of Eq.(2) can exist, of which two are stable to fluctuations in $E_{r}$. One, the 'ion root,' in which ions are held in by the electrons, is the one originally[6] and more commonly considered. At the second, 'electron root', the electrons are held in by the ions, and the particle and heat fluxes can be substantially reduced from those in the ion root. Here we show that the version of NHH2 studied here should be able to access this root. MHH2 is expected to display qualitatively similar behavior. Further reduction of nonaxisymmetric neoclassical transport is useful only when it is larger than the rates of both symmetric 
neoclassical and turbulent transport. Thus, in Sec. 5 we present some summarizing discussion of the results of earlier sections, including a comparison of the transport rates for these three mechanisms, with the turbulent rate estimated from some common empirical stellarator confinement scalings.

\section{Fields, Orbits, and Existing Theory}

In Figs. 1 we plot $B(\mathbf{x})$ along a field line for one poloidal transit for $\mathrm{MHH} 2$ (1a) and NHH2 (1b) on the flux surface $r=a / 2$. As noted in Sec. 1, one sees that the ripple amplitude $\delta$ is smaller than the slower toroidal variation $(\sim \epsilon)$. One notes that while $\mathrm{MHH} 2$ and NHH2 have precisely the same ripple amplitudes $B_{m n}$, the smaller $L_{r}$ for NHH2 noted in Sec. 1 makes its ripple wells much better defined and deeper than for MHH2. Absent in Fig. 1 and throughout this paper are those higher $n B_{m n}$ representing the discrete nature of the field coils, which are not computed by the fixedboundary VMEC calculations used here, and which should fall off rapidly as one moves inward in $r$ from the coils. Additionally, the remaining hundreds of harmonics which are produced by VMEC are ranked by size, and only the largest $N_{h}$ are kept. In Fig. $1 N_{h}=10$ harmonics are kept, which appears to capture most of the essential physics for the GC motion. Henceforth, it is convenient to denote the $(m, n)$-pairs by $(m, \tilde{n} \equiv n / N)$. The $(m, \tilde{n})$ s of NHH2's largest harmonics in descending order (those for MHH2 obtained simply by taking $m \rightarrow-m$ as noted earlier) are then $(0,0),(1,0),(2,-2)$, $(2,0), \ldots$ and the tenth largest $(1,1) . B_{m \tilde{n}}=B_{00} \equiv B_{0,0}$ gives the flux-surface averaged field, $B_{1,0} /\left.B_{00}\right|_{r=a} \simeq-.127$ yields the dominant $-\epsilon \cos \theta$ toroidal component of $B$, and $B_{2,-2} / B_{00} \simeq-.028$ is the largest nonaxisymmetric 
contribution. The smallest $B_{m \tilde{n}}=B_{1,1}$ kept there is down by about 2 orders of magnitude from $B_{0,0}$, and a factor of 4 from $B_{2,-2}$.

The general features of orbits in these fields are as envisioned in the established literature on particle motion and neoclassical transport in stellarators. In Figs. 2 and 3 we show poloidal projections of some collisionless orbits representative of those dominantly contributing to neoclassical transport in $\mathrm{NHH} 2$, for three values of the ambipolar field, given by the dimensionless variables $\hat{\phi} \equiv R_{0} e_{i} E_{r} / E_{\alpha}$ or $\phi \equiv a e_{i} E_{r} / T_{i}$ (with $E_{\alpha}=3.5$ $\mathrm{MeV}$ the alpha birth energy, and $T_{i}$ the ion temperature, which we take as $3.5 \mathrm{keV}=E_{\alpha} / 10^{3}$.): (a) $\hat{\phi}=-.01(\phi=-2.2)$, (b) $\hat{\phi}=0(\phi=0)$, (c) $\hat{\phi}=.01$ $(\phi=2.2)$. All are launched with kinetic energy $K=2 T=7 \mathrm{keV}$ at $\theta=0=\zeta$ and at small pitch $\lambda \equiv v_{\|} / v$. Those in Fig. 2 have $\lambda=0$ and an initial phase which makes them ripple trapped, while those in Fig. 3 have $\lambda=.2$, which are toroidally trapped, like normal tokamak bananas.

For $E_{r}=0$, trapped particles [Fig. 2(b)] drift directly out of the machine. For nonzero $E_{r}$, these 'superbanana' orbits acquire a poloidal drift which for $\phi \sim 1$ is large enough that the superbananas are well confined. While particles initially ripple trapped sometimes remain trapped for the entire poloidal transit [Fig. 2(c)], a more common situation is illustrated by Fig. 2(a), where a particle initially ripple trapped can make successive collisionless transitions from and back to that trapping state. Analytic theory of this process $[7,8]$ assumes that sufficient symmetry in $B(\mathrm{x})$ exists that the collisionless superbananas approximately close on themselves. This characteristic is only roughly satisfied for $\mathrm{NHH} 2$, so that one might expect existing theory to capture much of the transport physics here, though not all. Owing to the larger $L_{r}$, collisionless orbits in MHH2 display less symmetry, with 
collisionless de- and re-trapping being more frequent and less regular.

The orbits in Figs. 3 are tokamak-type bananas. Due to the ripple perturbations $(n \neq 0)$, these bananas drift radially. $E_{r}$ affects the size of the bananas, as visible in Fig. 3, as well as the toroidal precession rate, and thereby the size of the radial excursion these drifting bananas make, giving the $E_{r}$-dependence in the "banana-drift branch" $(b=b d)$ of transport. $[9,10]$ In the absence of the $n \neq 0$ harmonics, bananas close on themselves, producing the standard axisymmetric transport $(b=s y m)$ of an ideal tokamak. Finally, the ripple trapped particles illustrated in Fig. 2 produce the "superbanana branch" ( $b=s b$ ) of transport. The transport coefficients $D^{b}$ for each of these 3 branches $b=s y m, b d, s b$ are plotted in Fig. 4 for the present $\mathrm{MHH} 2$ parameters. As a rough but standard approximation, to estimate the total transport expected from a system in which all three transport mechanisms are operative, we shall take the sum of the contributions from the three individual branches.

Each $D^{b}$ has various collisionality regimes, denoted by the subscripts. $D^{s y m}$ has the familiar banana, plateau, and Pfirsch-Schlïter regimes (the last beginning at higher central electron density $n_{e 0}$ than the range shown in Fig. 4) $D_{b n, p l, p s}^{s y m}$, scaling with collision frequency $\nu \propto n_{\mathrm{e} 0}$ as $D_{b n, p s}^{s y m} \sim \nu$ and $D_{p l}^{s y m} \sim \nu^{0} . D^{b d}$ has the less and more collisional banana-drift regimes[9] $D_{1 E,-1}^{b d}$, scaling with $\nu$ and poloidal $E \times B$ precession frequency $\Omega_{E} \propto \phi$ as $\nu^{1} / \Omega_{E}^{2}$ and $\nu^{-1} \Omega_{E}^{0}$, and the still more collisional ripple-plateau regime[10] $D^{b d} \sim \nu^{0} \Omega_{E}^{0}$. $D^{s b}$ has the least collisional "superbanana regime" $[7,8] D_{1 E}^{s b} \sim$ $\nu^{1} / \Omega_{E}^{2}$ where trapping and detrapping effects are significant, an intermediate " $\nu^{1 / 2}$ regime" [6] $D_{1 / 2}^{s b} \sim \nu^{1 / 2} / \Omega_{E}^{3 / 2}$ whose width in $\nu$ or density vanishes for $\delta<\epsilon$, as here, and the " $1 / \nu$ regime" $D_{-1}^{s b} \sim \nu^{-1} \Omega_{E}^{0}$, probably the most 
commonly used expression for stellarator ripple transport. The expressions plotted in Fig. 4 are only the asymptotic forms valid in each collisionality regime; a fuller treatment would round off the abrupt transitions which appear in Fig. 4. A second rounding effect occurs when the monoenergetic results plotted here are averaged over a Maxwellian distribution, as will be done in. Sec. 4.

\section{Numerical Results}

As noted in Sec. 1, the numerical transport coefficients $D$ presented in this section were computed from MC simulations using GC3, a GC code in Boozer coordinates, using numerical equilibria generated using VMEC and translated to Boozer coordinates using the JMC code.[2] GC3 uses a Lorentz collision operator, and each value of $D$ was computed from a monoenergetic ensemble of 352 particles launched at $r / a=.5$, with $x \equiv K / T=2$, and evenly distributed in initial values of $\theta, \zeta$, and $\lambda$. D is calculated from the Fokker-Planck form

$$
D=\left\langle(\delta r)^{2} /(2 \tau)\right\rangle
$$

where $\langle\ldots\rangle$ is an ensemble average, $\delta r(\tau) \equiv r(\tau)-\langle r\rangle$, and $\tau$ is the orbit run time. To yield a genuine diffusion coefficient, $\tau$ must be longer than a radial decorrelation time, indicated by a transition to a behavior $\left\langle(\delta r)^{2}\right\rangle \sim \tau$. in a plot of $\left\langle(\delta r)^{2}\right\rangle$ versus $\tau$ as $\tau$ becomes long enough. This is satisfied for most of the numerical results shown here. However, for small $\phi$ and at low collisionality, trapped ions can walk nondiffusively out of the machine. When a particle escapes, $\tau$ is given by the run time for that particle, and $r(\tau)$ in $\delta r$ is given by $a$. When most particles contributing to the transport 
have nondiffusive motion, $D$ in Eq.(3) no longer has a strict interpretation as a diffusion coefficient, but still is an average reciprocal confinement time[11] (times a constant), and so is still a good measure of the confinement quality of the machire.

In Fig. 5 are shown numerical and analytic transport coefficients for NHH2 versus density $n_{e 0}$ (or $\nu$ ), for $\hat{\phi}=0$. The 4 curves with symbols are numerical results, and the 4 heavier curves without symbols are analytic ones. The 3 lower analytic curves show each of the 3 transport branches, and the top curve is their sum. The curve with diamond symbols shows MC results taking $N_{h}=2$, i.e., taking only the two largest harmonics $(0,0)+(1,0)$, thus simulating the ideal tokamak nearest to the full MHH2 system. This latter is approximated by the curve labeled $N_{h}=10$ (star symbols). First comparing the $N_{h}=2$ curve with $D^{s y m}$, one notes approximate agreement between the numerical diffusion and the axisymmetric neoclassical prediction. Additionally, comparing the $N_{h}=10$ 'full' results with the total (solid) analytic curve, one again notes rough agreement. As $n_{e 0} \rightarrow 0$, the analytic curves become infinite, with the onset of the $1 / \nu$ regime, while of course the numerical results remain finite, and represent transport which is no longer diffusive. As already noted, the NHH2 fields do not fully satisfy assumptions made in the original stellarator theories yielding the analytic expectations plotted here, and thus the approximate agreement in Fig. 5 is as good as one might expect.

Two other MC curves appear in Fig. 5, the results of two truncations of the full fields to test the contribution to the transport of particular $B_{m \tilde{n}}$ s. The curve labeled $N_{h}=3$ (rectangular symbols) shows the transport from keeping only the largest $n \neq 0$ harmonic $(2,-2)$ in addition to the two 
$(0,0)+(1,0)$ kept for the $N_{h}=2$-truncation. One sees that the $(2,-2)$ alone accounts for about half the ripple transport of the full $N_{h}=10$ system. The curve labeled $N_{h}=4$ (triangular symbols) gives the results of keeping only the $(0,1)+(0,-1)$ harmonics in addition to the $(0,0)+(1,0)$ axisymmetric ones. These yield a mirroring field which can be problematic for some stellarator configurations[12]. For the present case, however, one sees the transport contribution from this mirroring perturbation is rather small.

Fig. 6 shows a sweep of MC results versus $\hat{\phi}$ at $n_{e 0}=3 \times 10^{13} / \mathrm{cm}^{3}$, for the full $N_{h}=10$ and axisymmetric $N_{h}=2$ cases. For simplicity, the value for the ripple-strength $\delta$ required for the analytic curves is computed using only the largest contributor $B_{2,-2}$. This makes the width of the analytic peaks around $\hat{\phi}$ or $\Omega_{E}=0$ broader for both $D^{b d}$ and $D^{s b}$ than one actually expects as both mechanisms make the transition from their $1 / \nu$ to their $\nu / \Omega_{E}^{2}$ behavior, and one sees the the $N_{h}=10$ numerical results do indeed have the peaked form of the analytic curves, but fall off somewhat faster with $\hat{\phi}$.

In Fig. 7 we compare the simulation results for $\mathrm{NHH} 2$ already discussed with those for MHH2. One notes that while being of comparable size, the scaling of $D$ with $n_{e 0}$ is somewhat different for MHH2, and is not as close to the analytic prediction. Again, in general terms, this is expected, because of the closer adherence in NHH2 of the spatial separation $L_{t} / L_{r} \gg 1$. And qualitatively, the lower transport levels for low $n_{e 0}$ is probably due to the more prevalent collisionless detrapping enhancing the effective collision frequency in this regime. However, a fuller understanding of the transport scaling in $\mathrm{MHH} 2$ probably requires a theory different from the traditional ones considered so far. Some work has been done[13] generalizing the banana- 
drift branch to perturbations which, like those here, have low $-n$ and $m \neq 0$. Whether theory along these lines can clarify the MHH2 results in Fig. 7 is under study. For the present, however, both analytic and numerical indications are that one may take the more complete results given here for NHH2 as an estimate of the transport levels and scalings one may expect for $\mathrm{MHH} 2$.

\section{Energy Averaging and Ambipolar Potential}

We have seen that existing analytic theory for stellarator transport provides an approximate understanding of the monoenergetic simulation results for $\mathrm{NHH} 2$, though that configuration is somewhat different from the more 'classical' stellarators for which the theory was first developed. On this basis, we analytically develop an expectation for the fluxes one may expect from a local Maxwellian distribution $f_{M}$, and from these expressions, which depend on the ambipolar electric field $E_{r}$, obtain a solution for the expected ambipolar field and predictions for the particle and heat fluxes in the presence of that $E_{r}$. The general procedure is like that used earlier[6,4], but includes the two transport branches $b=s y m, b d$ in addition to the $s b$-branch considered in that earlier work.

For any function $g(x \equiv K / T)$ of the kinetic energy, the radial flux of $g$ for species $s$ due to transport branch $b$ is given by

$$
\gamma_{g s}^{b} \equiv \Gamma_{g s}^{b} /\left(n_{s 0} a\right) \equiv-\int d \mathbf{v} g(x) D_{s}^{b}(x) \partial_{r} f_{M s} /\left(n_{s 0} a\right)
$$

where the normalized flux $\gamma_{g s}^{b}$ is defined to have units of inverse time, so that the confinement time $\tau_{g}$ for $g$ is approximately given by $1 /\left(4 \gamma_{g}\right) . D_{s}^{b}(x)$ 
is the monoenergetic diffusion coefficient examined in Sec. 3. Specializing $g$ to $x^{r}$, for any power $r$, one may perform the velocity integration, finding[4]

$$
\gamma_{x^{r}}=\frac{2}{a^{2} \sqrt{\pi}}\left\{\bar{D}_{s}^{b}(r)\left[\left(a \kappa_{n}\right)+\phi_{s}-\frac{3}{2}\left(a \kappa_{T}\right)\right]+\bar{D}_{s}^{b}(r+1)\left(a \kappa_{T}\right)\right\}
$$

where $\kappa_{n} \equiv-\partial_{r} \ln n_{0}, \kappa_{T} \equiv-\partial_{r} \ln T, \phi_{s} \equiv a e_{s} E_{r} / T_{s}$, and

$\left.\bar{D}^{b}(r) \equiv \sum_{q} D_{q}^{b}(x=1) I\left(x, \frac{1}{2}+m_{q}+r\right)\right|_{-} ^{+}$is $D^{b}(x)$ averaged over energy, and hence over successive collisionality regimes $q$. The energy integral is given by the incomplete Gamma function $\Gamma(n+1, x) \equiv I(x, n) \equiv \int^{x} d x_{1} x_{1}^{n} e^{-x_{1}}$, and the limits $\left.\right|_{-} ^{+}$denote those values $x_{ \pm}$at which the collisionality regime $q$ of the transport mechanism changes. As mentioned in Sec. 2, the effect of performing this integration is to smooth the abrupt transitions in the transport coefficients plotted in Fig. 4. From Eqs. 4 or 5, the normalized particle flux is $\gamma_{1}$ (i.e., $r=0$ ), and the heat flux is $\gamma_{x}$ (i.e., $r=1$ ).

The symmetric contributions $\gamma^{\text {sym }}$ to the particle fluxes are intrinsically ambipolar, and thus do not play a role in determining $E_{r}$. Thus, in Fig. 8 are plotted the nonaxisymmetric portions $\gamma^{n a}=\gamma^{b d}+\gamma^{s b}$ of $\gamma$ versus $\hat{\phi}$, for both ions and electrons, along with the constituent parts $\gamma^{b d}$ and $\gamma^{s b}$. The point $\hat{\phi}_{1} \simeq-.01$ at which $\gamma_{1 i}^{n a}=\gamma_{1 e}^{n a}$ is a self-consistent solution for $E_{r}$ at the single radius $r_{0}=a / 2$ for which this calculation has been carried out. $\hat{\phi}=\hat{\phi}_{1}<0$ is the 'ion root' more commonly considered. For the present parameter choice, the second 'electron root' $\hat{\phi}_{2}>0$ of the ambipolarity constraint (2) does not occur, essentially because the ion flux or diffusion coefficient does not fall off rapidly enough above $\hat{\phi}=0$ to drop below the slowly declining electron flux $\gamma_{1 e}$ before it falls below 0 due to $\phi_{e}$ in Eq.(5).

Plotting the full energy fluxes $\gamma_{x s}$ versus $\hat{\phi}$, and superposing the value $\hat{\phi}=\hat{\phi}_{1}$ established from Fig. 8, in Fig. 9 one reads off the expected ion and 
electron heat fluxes for that case, and from this in Fig. 10 the predicted neoclassical ion and electron energy confinement times $\tau_{E s} \equiv \tau_{x s},(s=i, e)$, finding $\tau_{E i} \simeq 44 \mathrm{~ms}$, and $\tau_{E e} \simeq 68 \mathrm{~ms}$.

The width of the peak in $\gamma_{i}^{n a}$ is proportional to collisionality. Thus, one way of accessing the electron root for this system would be to operate at lower density (or higher temperature). (A more precise criterion is given in Ref. 4.) This is illustrated in Fig. 11, where the density is lowered by a factor of 3 , to $n_{e 0}=10^{13}$. Now the electron root does appear, at $\hat{\phi}_{2} \simeq .03$, while the ion root is at $\hat{\phi}_{1} \simeq-.004$. One notes the property of $\hat{\phi}_{2}$ making it most interesting: While at $\hat{\phi}_{1}$ the fluxes are $\gamma_{1}^{n a} \simeq 1.9 / \mathrm{sec}, \gamma_{x i}^{n a} \simeq 12.0 / \mathrm{sec}$, and $\gamma_{x e}^{n a} \simeq 3.5 / \mathrm{sec}$, at $\hat{\phi}_{2}$ these are substantially reduced: $\gamma_{1}^{n a} \simeq .26 / \mathrm{sec}, \gamma_{x i}^{n a} \simeq$ $1.6 / \mathrm{sec}$, and $\gamma_{x e}^{n a} \simeq 1.6 / \mathrm{sec}$

\section{Discussion}

The NHH2 system employed in this work provides a bridge between MHH2, which is the configuration of principal interest, for which adequate analytic theory does not yet exist, and the more idealized existing analytic theory. It is noteworthy that 2 systems having identical descriptions except for the mapping $m \rightarrow-m$ which distinguishes them, can have rather different transport scalings. However, we have also found that the overall transport levels from the 2 systems are similar, so that our more detailed understanding of NHH2's transport can be used to estimate that for MHH2.

Tailoring the harmonic content of the $B_{m \tilde{n}}$ s to further reduce $\gamma^{n a}$ might be worthwhile for MHH2 if $\gamma^{n a}$ were large enough to dominate the symmetric neoclassical contribution $\gamma^{s y m}$ as well as the anomalous (turbulent) 
contribution $\gamma^{a n}$. To estimate the last of these, here we evaluate some common empirical stellarator scaling laws. However, it should be kept in mind that, as recent tokamak experiments with transport barriers show, turbulent transport is also a mechanism which may be amenable to substantial reduction.

The nonaxisymmetric neoclassical contributions $\gamma^{\text {na }}$ to the fluxes are comparable to the symmetric ones, and for NHH2 $\gamma^{n a}$ is dominated by $\gamma^{s b}$. At the ambipolarity solution $\hat{\phi}=\hat{\phi}_{1}$, for example, one finds $\gamma_{x i}^{\text {sym }} \simeq$ 2.93, $\gamma_{x i}^{s b} \simeq 2.23, \gamma_{x i}^{b d} \simeq 0.24$. Most ions are in the ripple-plateau regime of $\gamma^{b d}$, which has an $n$-scaling $D_{r p}^{b d} \sim n$. Thus, since for the present system $n \sim 4$ is small compared with that $(n \sim 20)$ producing TF ripple in a normal tokamak, $\gamma^{b d}$ is smaller by about a factor of 5 than its value for a normal tokamak with comparable ripple, making the banana-drift contribution subdominant.

Turning to the comparison of $\gamma^{n a}$ with $\gamma^{a n}$, we evaluate the confinement expected from the Lackner-Gottardi[14] and International Stellarator Scaling[15] expressions. As in Ref. 15, the units here are MKS, except for line-averaged density $\bar{n}_{19}$, which is in units of $10^{19} / \mathrm{m}^{3}=10^{13} / \mathrm{cm}^{3}$, heating power $P_{M W}$, which is in $\mathrm{MW}$, and $T_{e V}$, which is in $\mathrm{eV}$.

$$
\begin{aligned}
\tau_{E}^{L G} & =0.17 R a^{2} \bar{n}_{19}^{.6} B^{.8} q^{-.4} P_{M W}^{-.6} \\
\tau_{E}^{I S S 95} & =.079 R^{.65} a^{2.21} \bar{n}_{19}^{.51} B^{.83} q^{-.4} P_{M W}^{-.59} .
\end{aligned}
$$

Combining either of these with the power balance relation

$$
\tau_{E} \simeq 2 \pi^{2} R a^{2} \bar{n} T / P \simeq\left(.316 \times 10^{-4}\right) \bar{n}_{19} T_{e V} / P_{M W}
$$


yields a relation for $\tau_{E}$ as a function of $R, a, B, q$, and $\bar{n}_{19}$. For $\bar{n}_{19}=3$, one finds $\tau_{E}^{L G} \simeq 522 \mathrm{msec}$, while $\tau_{E}^{I S S 95} \simeq 21 \mathrm{msec}$, with $\tau_{E}^{L G}$ independent of $\bar{n}_{19}$, and $\tau_{E}^{I S S 95}$ only weakly dependent on $\bar{n}_{19}$.

Thus, if $\tau_{E}^{I S S 95}$ is assumed an accurate measure of turbulent transport, that transport dominates the neoclassical transport computed earlier, and further transport optimization from the configurations studied here would be of no use. On the other hand, if $\tau_{E}^{L G}$ is taken as proper measure, then the transport would be neoclassically dominated. However, since $\gamma_{x i}^{\text {sym }} \sim$ $\gamma_{x i}^{n a}$, as noted above, even complete elimination of $\gamma^{n a}$ would only result in modest gains in the total $\tau_{E i}$. Thus, further effort at optimization of thermal transport from the configuration adopted here seems of limited utility.

While thermal transport is thus acceptably low in the present configurations, it is strongly dependent on the radial field $E_{r}$ for being so, with $\gamma_{x i}$ reduced a factor of $\sim 8$ from its $\hat{\phi}=0$-value at the operating point $\hat{\phi}_{1}$. Thus, these systems will have a loss region for energetic ions. The existence of this loss channel has sometimes been regarded as a virtue,[16] providing a built-in means of alpha ash removal in return for a tolerable level of loss. If the loss rate is judged excessive, however, little optimization has been attempted on the configuration, and it seems likely that the loss could be further reduced with additional effort at tailoring the magnetics.

\section{Acknowledgment}

This work supported by U.S.Department of Energy Contract No.DEAC02-76-CHO3073. 


\section{References}

[1] P.R. Garabedian, Phys. Plasmas 3, 2483 (1996).

[2] L.-P. Ku, A.H. Reiman, (private communications, 1996).

[3] S.P. Hirshman, J.C. Whitson, Phys. Fluids 26, 3553 (1986).

[4] H.E. Mynick, W.N.G. Hitchon, Nucl. Fusion 23, 1053 (1983).

[5] D.E. Hastings, W.A. Houlberg, K.C. Shaing, Nucl. Fusion 25445 (1985).

[6] A.A. Galeev, R.Z. Sagdeev, H.P. Furth, M.N. Rosenbluth, Phys. Rev. Letters 22, 511 (1969).

[7] A.A. Galeev,R.Z. Sagdeev, Sov. Phys. Usp. 12, 810 (1970).

[8] H.E. Mynick, Phys. Fluids 26, 2609-2615 (1983).

[9] R. Linsker, A.H. Boozer, Phys. Fluids 25, 143 (1982).

[10] A.H. Boozer, Phys. Fluids 23, 2283 (1983).

[11] H. Wobig, Z. Naturforsch. 37a, 906 (1982).

[12] J. Nührenberg (private communications, 1996).

[13] H.E. Mynick, Nucl. Fusion 26, 491 (1986).

[14] K. Lackner, N.A.O. Gottardi, NF 30, p767 (1990).

[15] U. Stroth, Murakami, R.A. Dory, H. Yamada, F. Sano, T. Obiki, National Institute for Fusion Science Report NIFS-375 (1995).

[16] D.D.-M. Ho, R.M. Kulsrud, Phys. Fluids 30, 442 (1987). 


\section{Figures}

Fig. 1. Plot of magnetic field strength $B(\mathbf{x})$ along $\mathbf{B}$ for one poloidal transit at $r=a / 2$ in (1a) MHH2, (1b) NHH2.

Fig. 2. Collisionless GC orbits launched with $\lambda=0, \theta=0=\zeta$, kinetic energy $K=7 \mathrm{keV}$, and for 3 values of the ambipolar field $(\mathrm{a}) \hat{\phi}=-.01$, (b) $\hat{\phi}=0$, and (c) $\hat{\phi}=.01$.

Fig. 3. As Fig. 2, but with initial $\lambda=0.2$.

Fig. 4. Analytic predictions for transport coefficients $D^{b}$ for each of the three operative transport branches $b=s y m, b d, s b$, for $|\hat{\phi}|=.002$ and a monoenergetic distribution with $x \equiv K / T=2$, or $\hat{x} \equiv K / E_{\alpha}=.002$.

Fig. 5. Numerical (curves with symbols) and analytic (heavier curves without symbols) transport results versus $n_{e 0}$ for $\hat{\phi}=0$. See text for details.

Fig. 6. Transport results versus $\hat{\phi}$ for $n_{e 0}=3 \times 10^{13} / \mathrm{cm}^{3}$. See text for details.

Fig. 7. $D$ versus $n_{e 0}$ for $\hat{\phi}=0$, comparing transport in MHH2 with NHH2. As in Fig. 5, the analytic theory is shown by curves without symbols.

Fig. 8. Plots of $\gamma_{1 s}^{n a}=\gamma_{1 s}^{b d}+\gamma_{1 s}^{s b}$ and $\gamma_{1 s}^{b d}$ and $\gamma_{1 s}^{s b}$ versus $\hat{\phi}$, for $s=e, i$ and at $n_{e 0}=3 \times 10^{13}$.

The point $\hat{\phi}_{1}$ where $\gamma_{1 i}^{n a}=\gamma_{1 e}^{n a}$ is a self-consistent solution for the ambipolar field $E_{r}$.

Fig. 9. Plot of $\gamma_{x s}$ for $s=i$, e versus $\hat{\phi}$, for the same parameters as in Fig. 8 . 
Fig. 10. Plot of energy confinement times $\tau_{E s}$ for $s=i, e$, for the same parameters as in Figs. 8 and 9.

Fig. 11. Plot of $\gamma_{1 s}$ for $s=i, e$ versus $\hat{\phi}$, for the same parameters as in Fig. 8, except at lower density $\left(n_{e 0}=10^{1} 3\right)$, in order to access the electron root $\hat{\phi}_{2}$, as well as the ion root $\hat{\phi}_{1}$ present in Fig. 8 . 

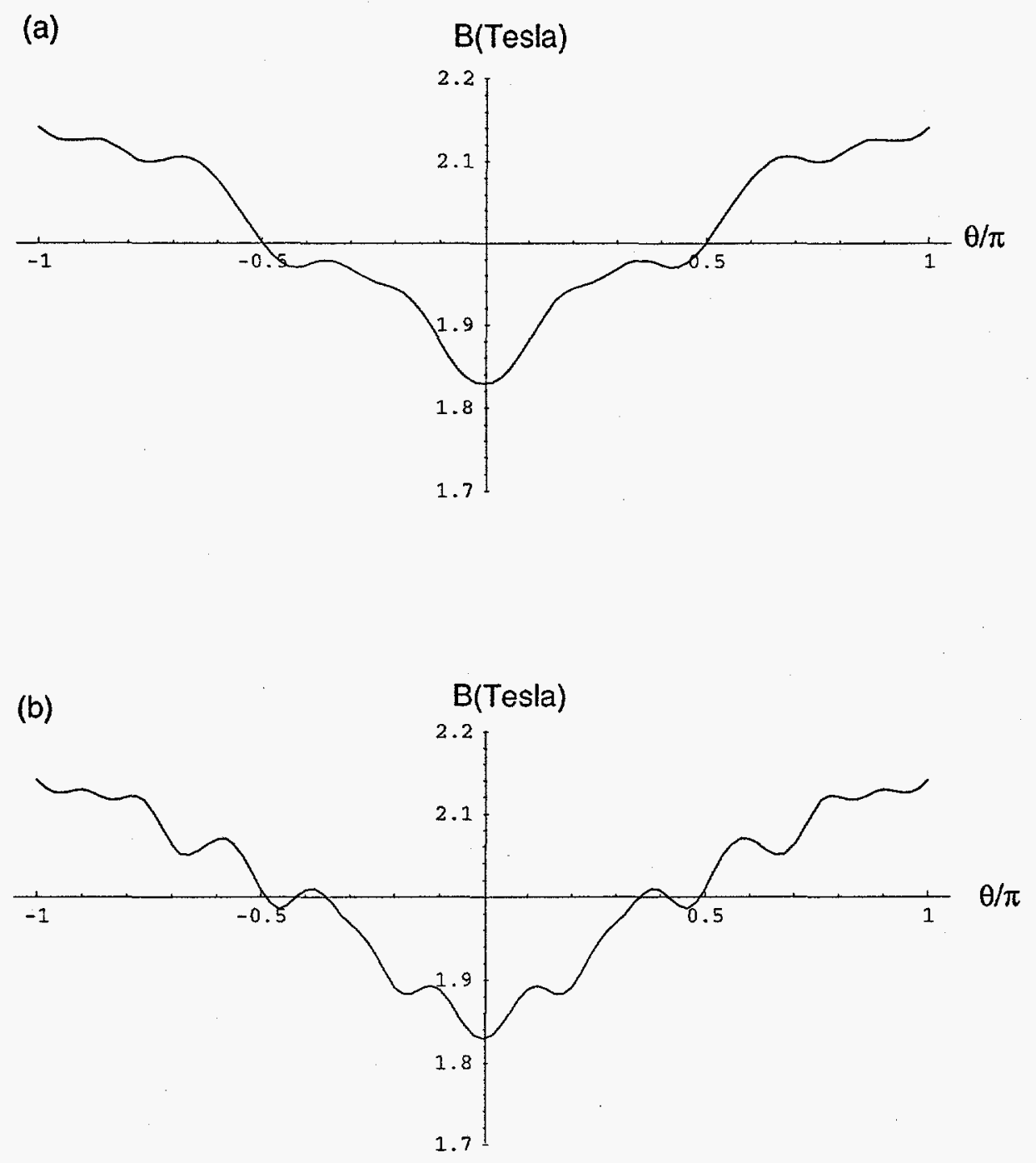

Figure 1: 

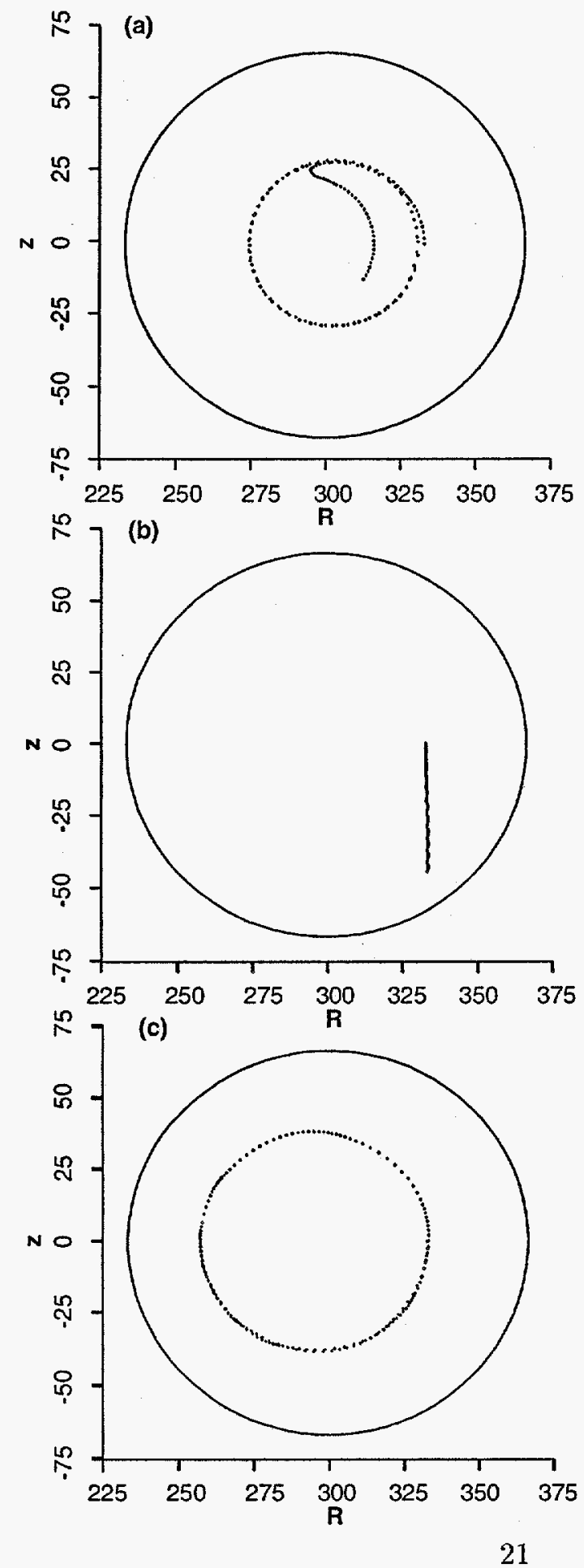

Figure 2: 

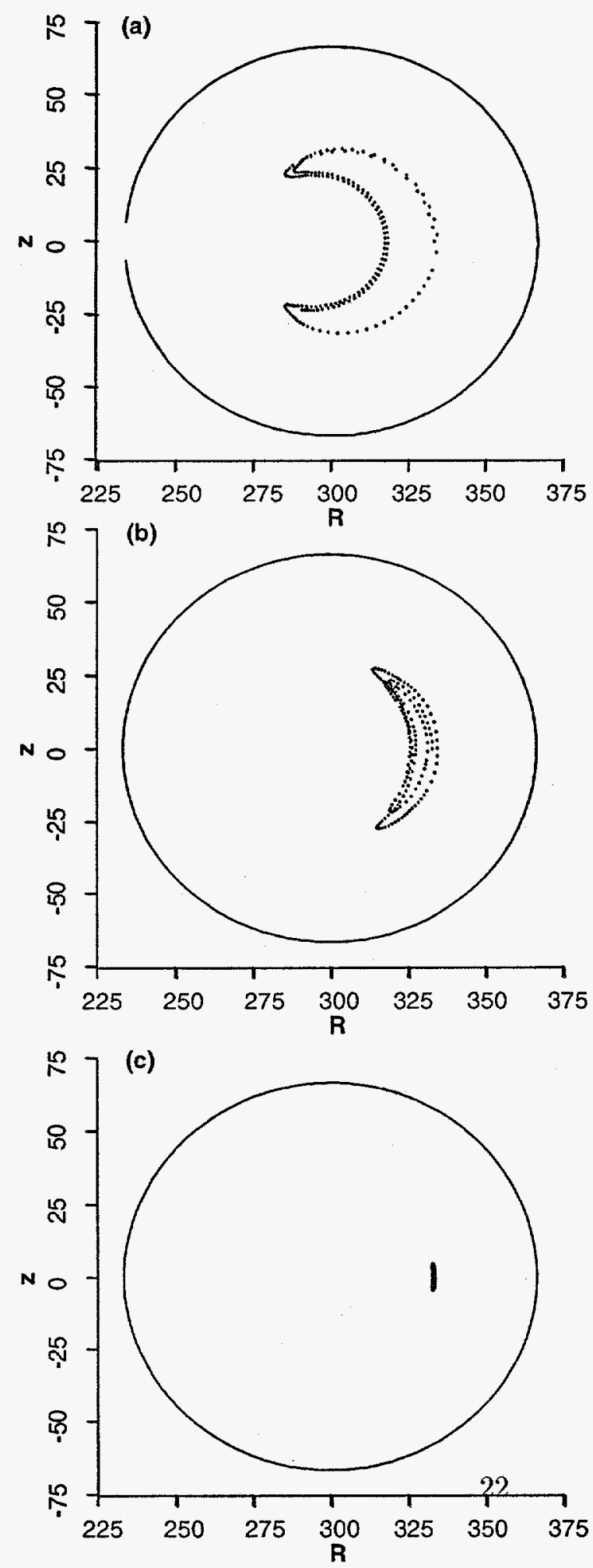

Figure 3: 


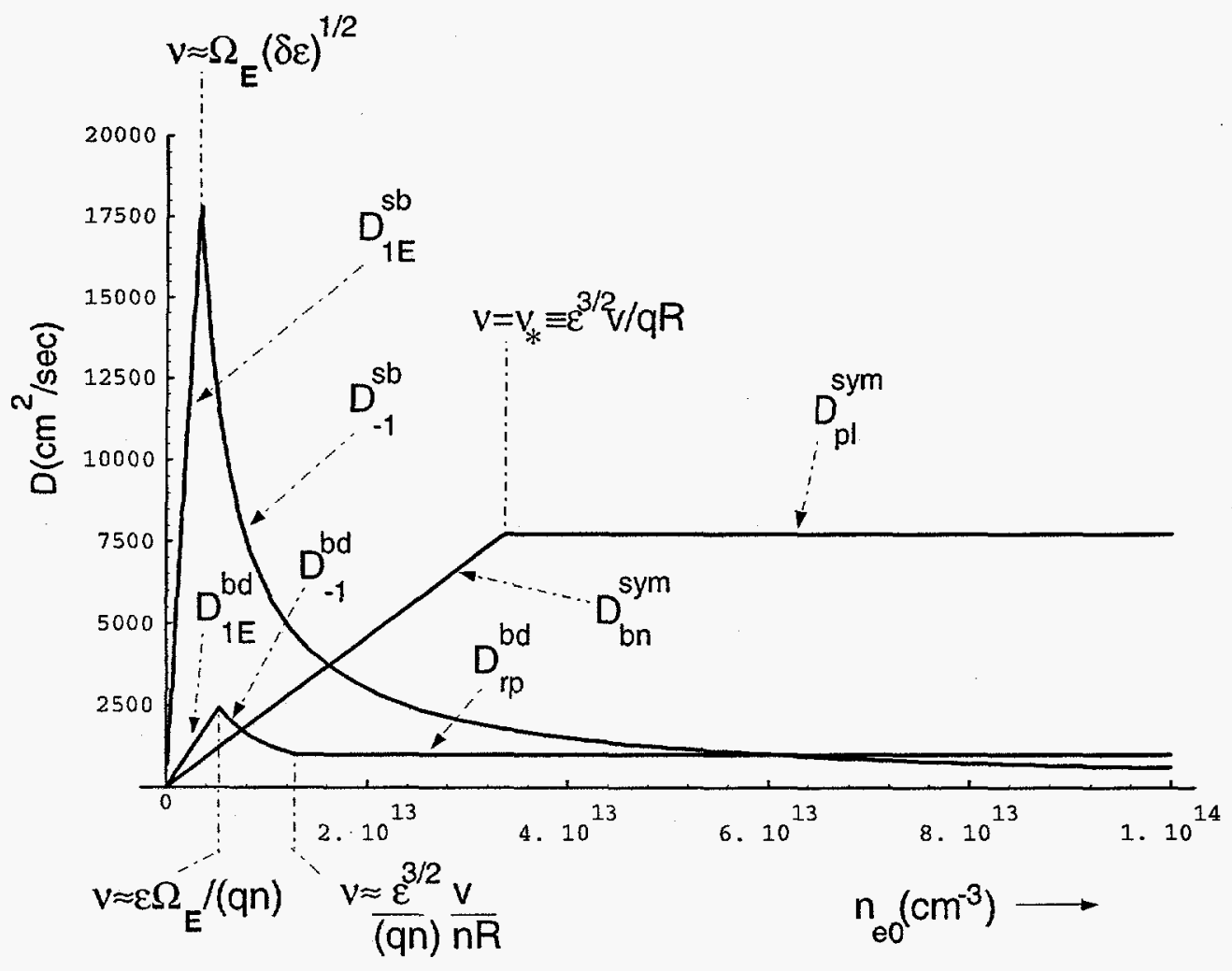

Figure 4: 


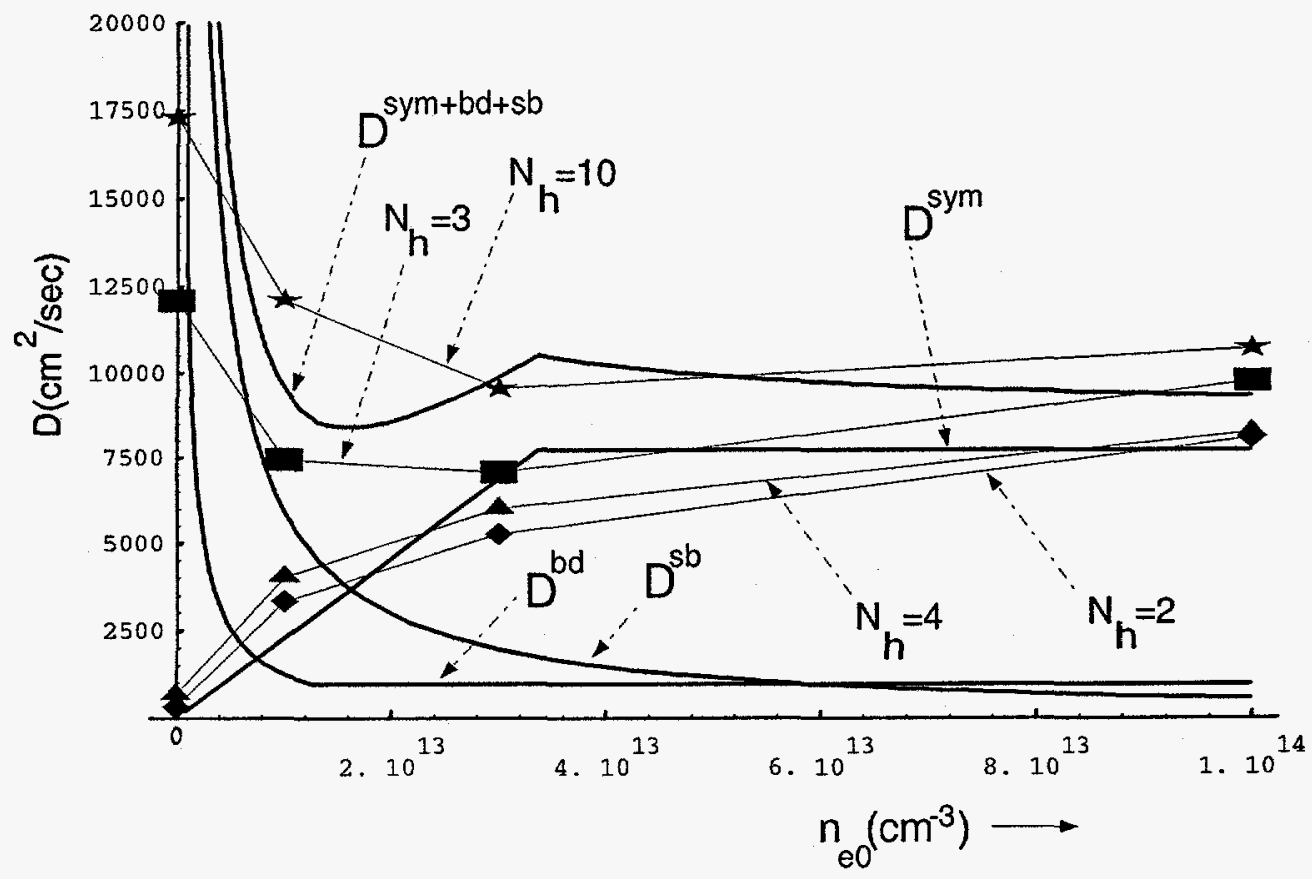

Figure 5: 


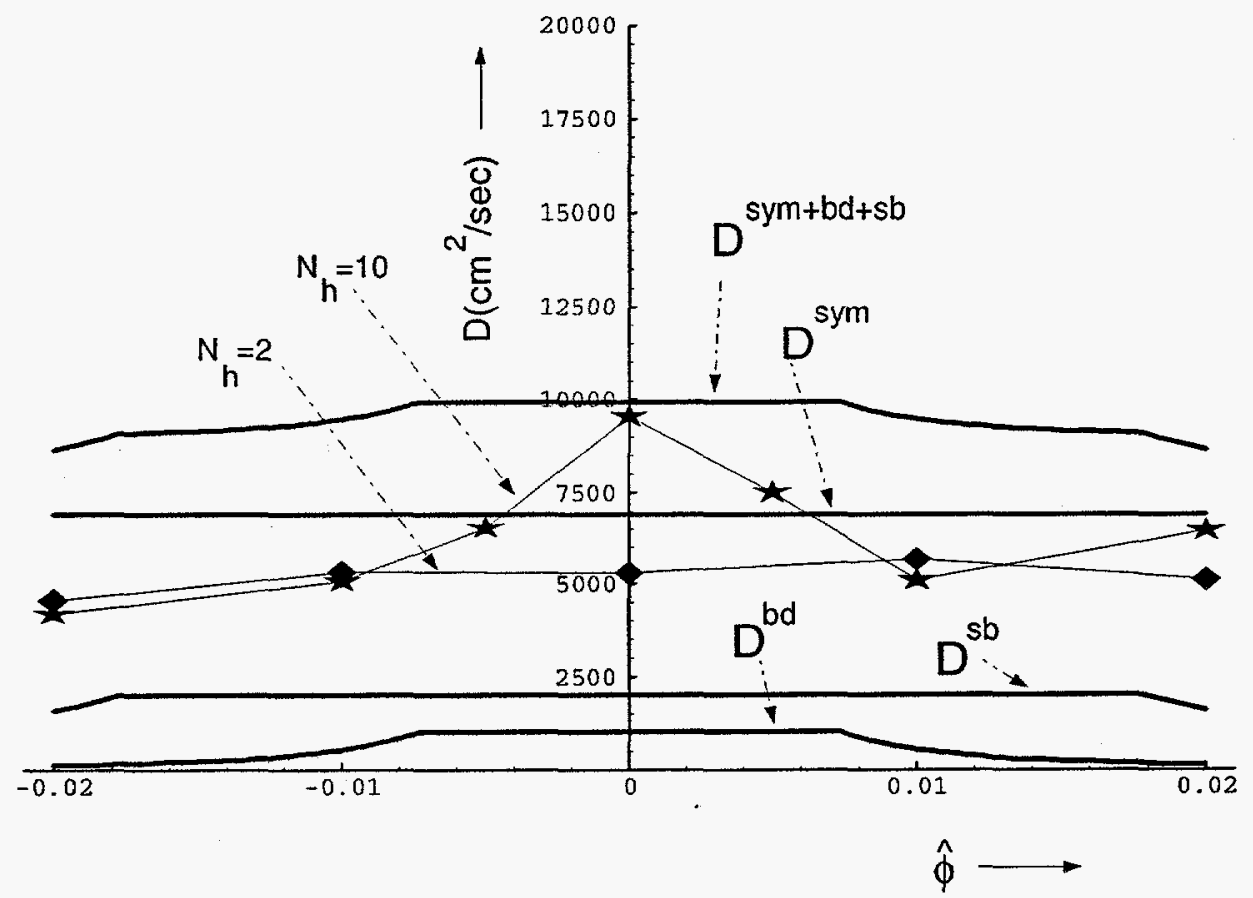

Figure 6: 


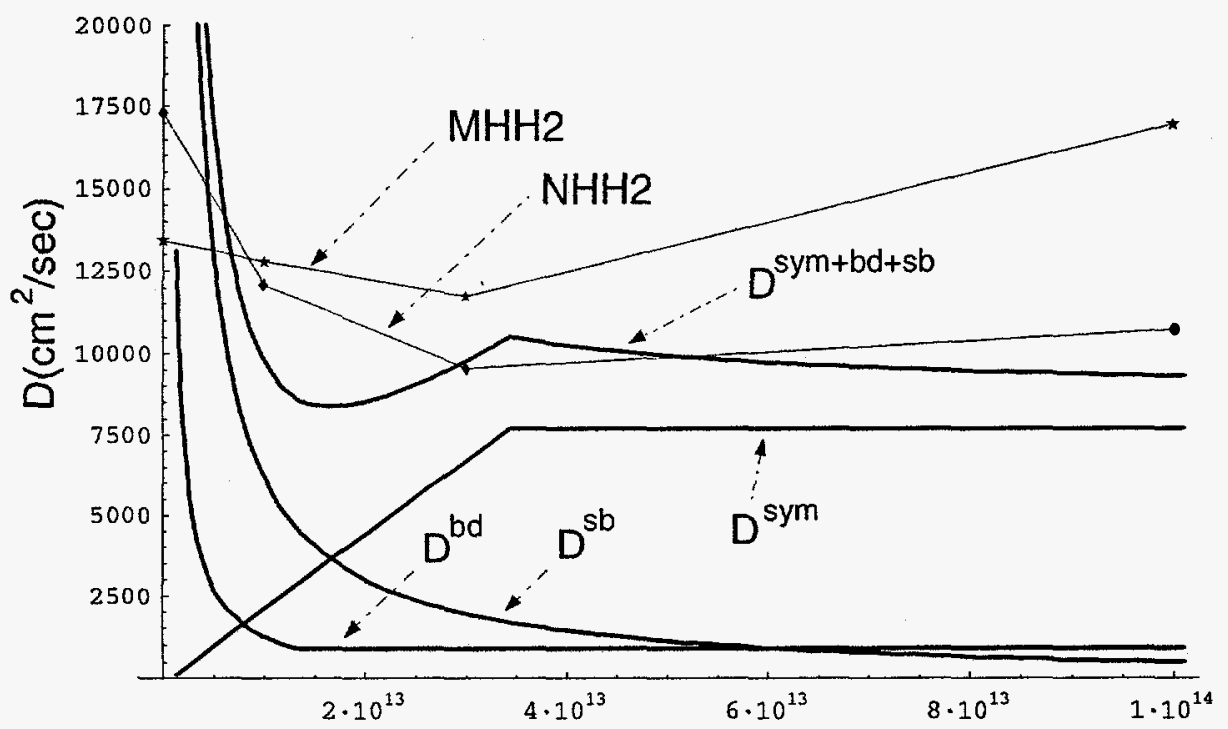

Figure 7: 


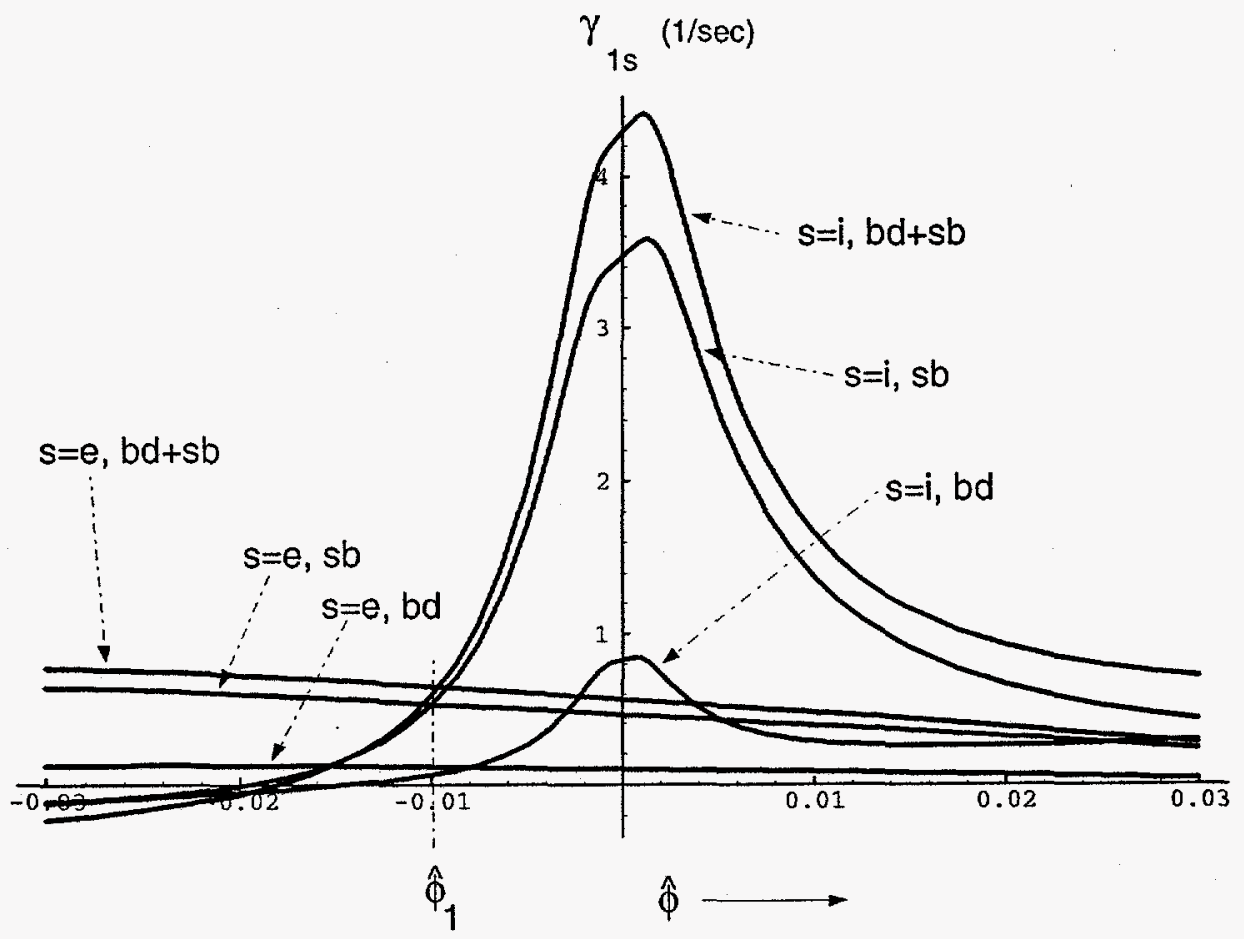

Figure 8: 


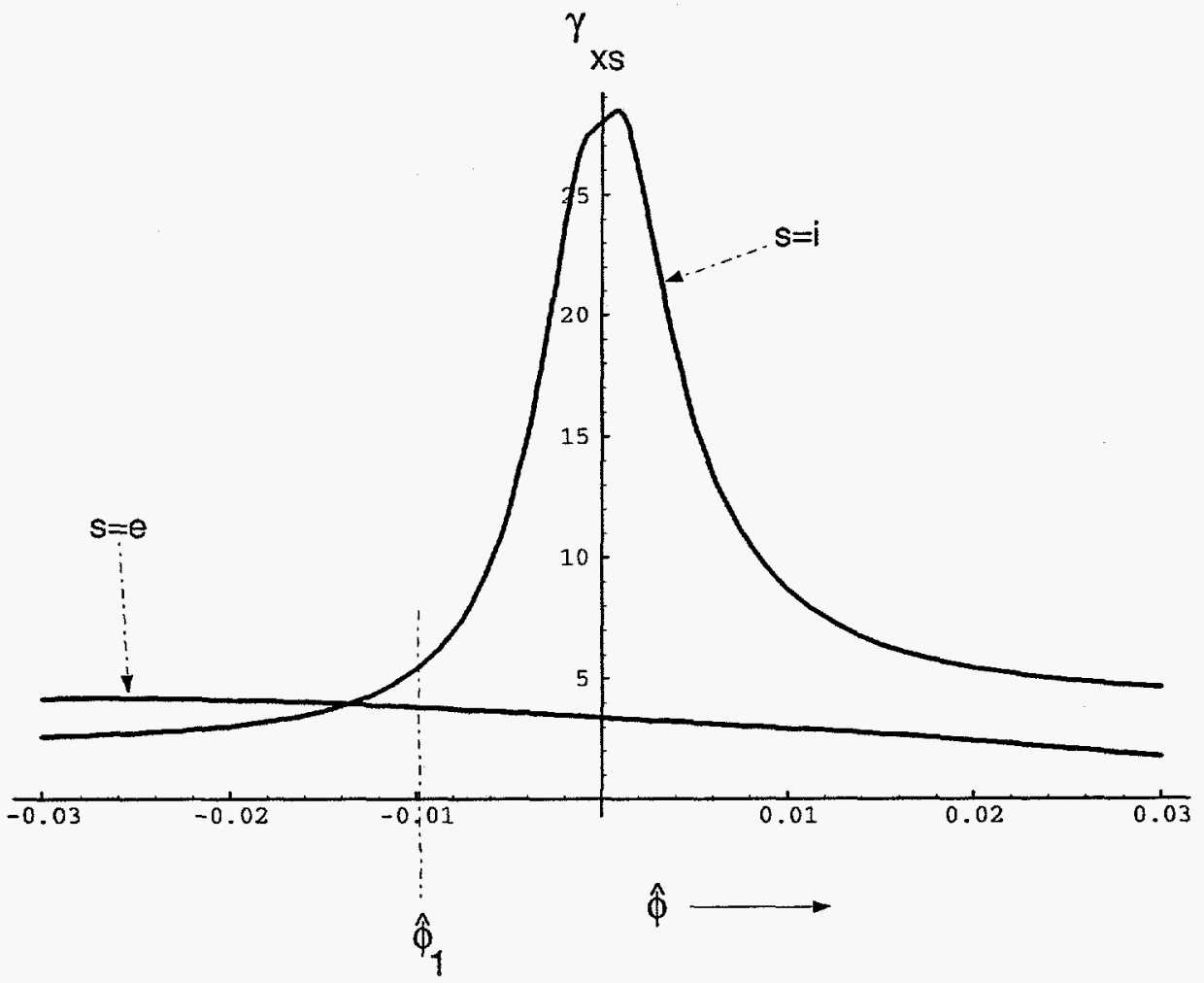

Figure 9: 


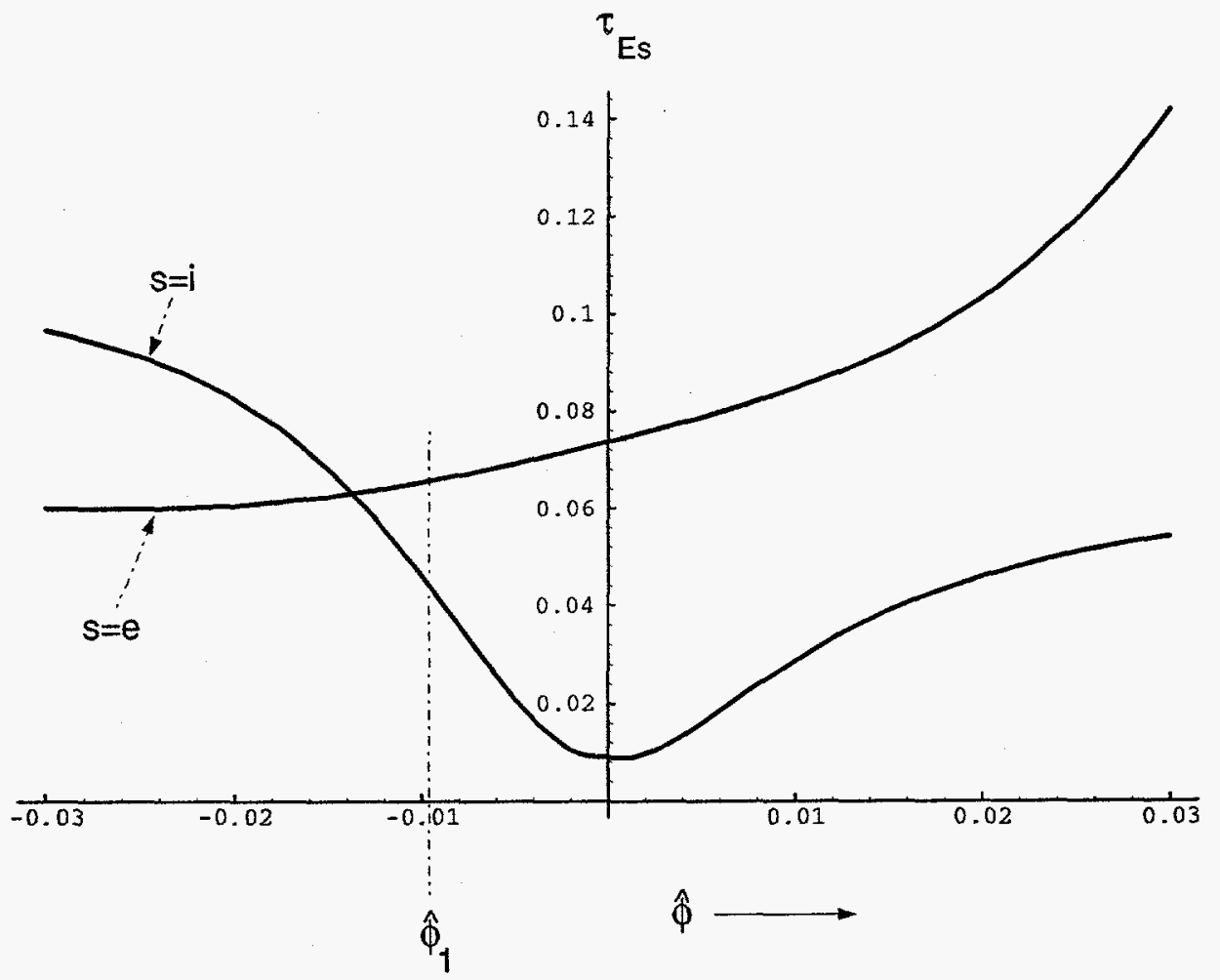

Figure 10: 


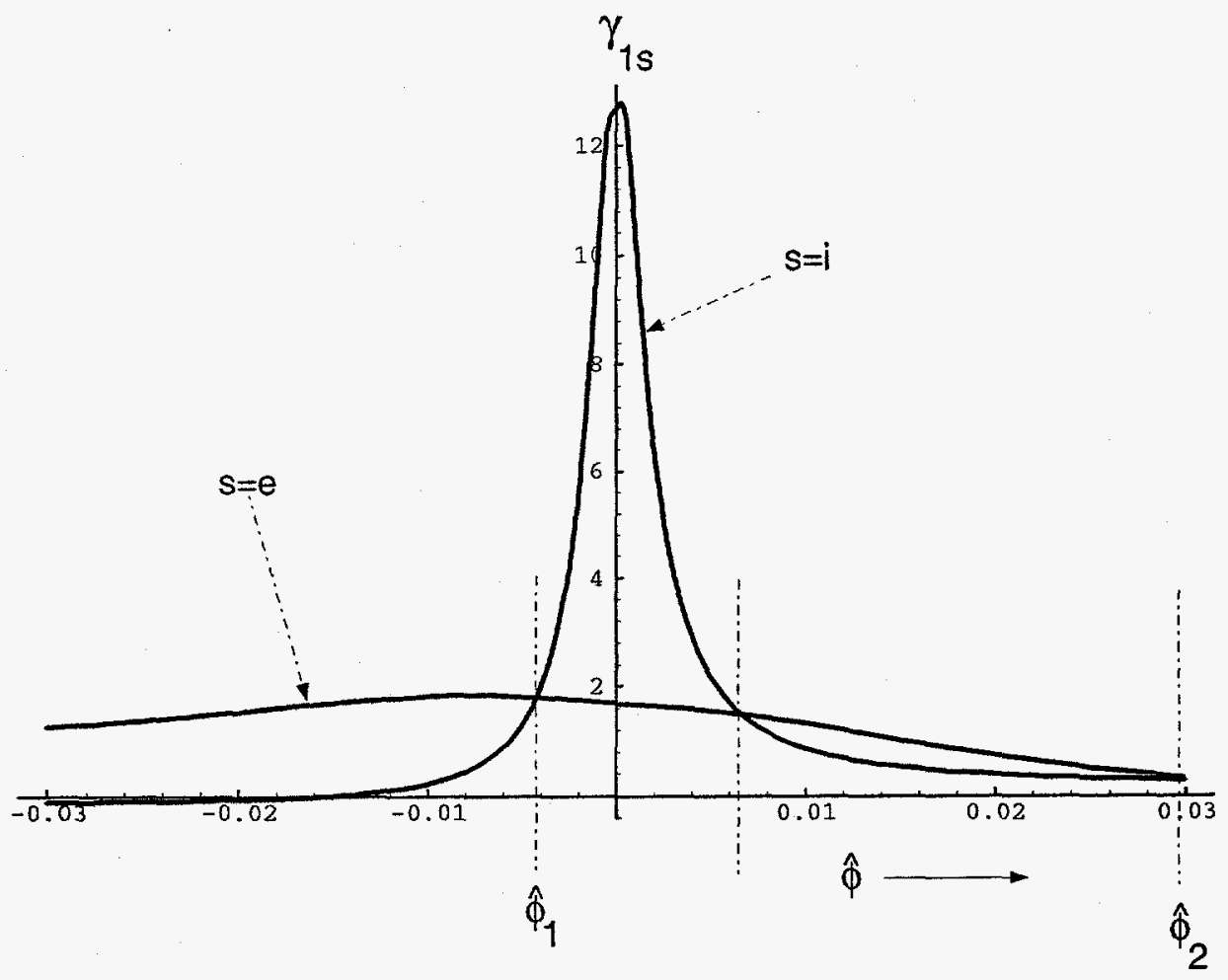

Figure 11: 